\title{
DIREITO À SAÚDE E À ATENÇÃO BÁSICA EM CAMPOS DOS GOYTACAZES, RJ, NO CONTEXTO DA PANDEMIA DA COVID-19*
}

\author{
Ana Paula Pessanha Cordeiro \\ Eliana Monteiro Feres \\ Valentina Sofía Suárez Baldo
}

\section{Introdução}

A pandemia ocasionada pelo coronavírus Sars-CoV-2, a Covid-19, responsável pela maior crise sanitária mundial da história, tem permitido várias reflexões. As incertezas causadas pela ausência de tratamento, somadas à falta de proteção, deixaram a população em situação de grande exposição. O Sistema Único de Saúde (SUS), que, desde sua criação, vem sendo sucateado pelo modelo neoliberal da gestão econômica no país, passou a ter defensores em todos os âmbitos, à medida que a pandemia ressaltou sua importância, por seu caráter público e universal.

Desse modo, o presente capítulo traz uma reflexão sobre a atenção básica no município de Campos dos Goytacazes, RJ, no contexto dessa crise de saúde, a partir da análise do Plano Municipal de Saúde de Campos dos Goytacažes 2018-2021 (PMS), tomando, como parâmetro, as diretrizes, os objetivos e as metas fixados por ele, principalmente os pontos que abordam o direito à saúde e a garantia de acesso à atenção de qualidade. Para tanto, consideram-se os contextos econômico, político e social da cidade nos últimos anos, o marco mais amplo da Política Nacional de Atenção Básica e as respostas dadas aos impactos da pandemia na cidade.

$\mathrm{Na}$ pesquisa, foram utilizados métodos de produção e de análise de dados de tipo qualitativo, a partir de fontes de informação primárias e secundárias: documentos oficiais da Prefeitura de Campos dos Goytacazes, bases de dados públicas do Instituto Brasileiro de Geografia e Estatística (IBGE) e do Conselho Nacional de Secretários de Saúde (Conass), além de material jornalístico, artigos, livros e dissertações.

As conclusões mostram uma defasagem entre o corpus considerado e os resultados obtidos até o presente, corroborando a existência de um projeto de desmonte do SUS e da proposta de atenção básica na cidade, situação que, ao contrário de ser isolada ou pontual, acompanha uma tendência de retrocesso da política de Saúde mais geral do país. Os resultados também evidenciam a necessidade de ações em defesa desse sistema público de saúde, mais urgentes do que nunca em tempos de pandemia.

Certamente a precarização e o sucateamento de saúde, na região, não se iniciaram com a pandemia, mas foram agravadas a partir dela. Refletir sobre o modelo de atenção adotado e sobre sua aplicação pelo gestor permite-nos apontar os impactos da falta de acesso à saúde e da não garantia do direito por parte do poder público.

${ }^{*}$ DOI- 10.29388/978-65-81417-27-7-0-f.147-163 


\section{O município de Campos dos Goytacazes}

Campos dos Goytacazes, município da região Norte Fluminense do estado do Rio de Janeiro, é uma cidade de longa história e de importante participação na produção da riqueza econômica nacional. É a quarta do estado com maior produto interno bruto (PIB), depois do Rio de Janeiro, de Duque de Caxias e de Niterói (IBGE, 2018). Em sua costa, encontra-se a maior bacia petrolífera do Brasil (PETROBRAS, 2021), descoberta na década de 1970, e que, junto ao Porto do Açu, inaugurado em 2014, contribuiu para a recuperação econômica recente da região. Na Bacia de Campos, são extraídos 80\% do petróleo nacional (PREFEITURA DE CAMPOS, 2018a), o que tem colocado o município em um lugar de destaque no cenário político e econômico do país. A descoberta do lençol petrolífero e as atividades de exploração que lhe seguiram implicaram a transformação da economia da cidade e promoveram sua modernização e desenvolvimento, na medida em que estimularam a ampliação da sua infraestrutura, a consolidação de políticas públicas, a atração de novas empresas e a geração de empregos (PREFEITURA DE CAMPOS, 2018a). Ramos (2016, p. 65) aponta que:

[...] a instalação da Petrobrás em Macaé na década de 70, e suas subsidiárias, prestadores de serviços e todas as atividades indiretas relacionadas ao crescimento do setor, irão trazer não só a contribuição dos repasses de royalties (que contribuem para gerar considerável acréscimo na receita municipal, tanto em termos diretos e indiretos) [...], mas uma ampla e diversificada gama de atividades que contribuem para a geração de empregos, circulação monetária, aumento de poder aquisitivo local e regional (ainda que guardadas importantes discrepâncias em termos de desnível social e de renda que se expressam, inclusive espacialmente) e aumento da circulação de pessoas a partir de uma nova, e peculiar, dinâmica migratória local e regional (RAMOS, 2016, p. 65).

O município ainda conta com extensão territorial de $4.032 \mathrm{~km}^{2}$, a maior do estado, ocupando quase 10\% da superfície deste (IBGE, 2020a). Compõe-se de quatorze distritos e é o mais populoso do interior (região que exclui os municípios da região metropolitana da cidade do Rio de Janeiro) (IBGE, 2020b), com uma população estimada de 511.168 habitantes (IBGE, 2020b). Nas últimas três décadas, Campos passou por um forte processo de urbanização (SILVA, 2012), sendo, atualmente, a segunda maior área urbana estadual, depois da cidade do Rio de Janeiro (FARIAS et al., 2017).

A cidade também conta com um índice de desenvolvimento humano considerado alto: passou de 0,505, em 1991, para 0,716, em 2010, o que implicou um crescimento de 41,78\%. Nesse último ano citado, no entanto, 10,19\% dos campistas estavam desempregados (ATLASBR, 2021), enquanto $37,7 \%$ da população vivia com renda de até meio salário-mínimo (IBGE, 2010). Nesse sentido, o Plano Municipal de Assistência Social de Campos dos Goytacažes 2018-2021 aponta que:

Os rebatimentos do processo de abolição da escravidão em Campos dos Goytacazes, uma das últimas cidades no Brasil a realizá-la, são sentidos até os dias atuais na medida em que esse contexto sem o desenvolvimento de um projeto econômico-social para essa população [...] resultou na reprodução de um ciclo da pobreza que até hoje perpassa gerações (PREFEITURA DE CAMPOS, 2018b, p. 15). 


\section{A pandemia da Covid-19 em Campos dos Goytacazes}

Em 11 de março de 2020, a Organização Mundial da Saúde (OMS) declarou como pandemia a doença causada pelo coronavírus Sars-CoV-2. A infecção, chamada de Covid-19, além de ser muito transmissível, tem letalidade estimada em cerca de 14 vezes mais que a da influenza. O alto índice de contágio, com cada indivíduo infectando de duas a três pessoas em média, causou a expansão da epidemia em progressão geométrica (DAUMAS, 2020). Pouco tempo depois do decreto da OMS, o mundo todo adotou medidas para evitar a disseminação da doença.

Fatores como a não existência de tratamento precoce eficaz para impedir a infeção, as incertezas iniciais sobre suas vias de contágio e o despreparo de um mundo integrado globalmente para lidar com o alastramento da pandemia, somados a uma acentuada escala de informações falsas sobre a natureza do vírus e da doença por ele produzida, em um contexto de sociedades altamente conectadas por meio de redes sociais, conduziram boa parte das nações do mundo à crise socioeconômica e sanitária.

O Brasil foi o primeiro país da América do Sul a identificar um caso confirmado de Covid-19, em 26 de fevereiro de 2020, na cidade de São Paulo. Depois desse momento, a doença proliferou-se de forma acelerada em todos os municípios. Em fins de maio de 2021, eram contabilizados mais de 16,5 milhões de ocorrências desde o início da pandemia, ultrapassando a triste marca de 462 mil mortes pelo novo coronavírus (CONASS, 2021). Nesse mesmo período, o Rio de Janeiro era o segundo estado do país com maior número acumulado de óbitos pelo vírus, registrando mais de 50 mil mortes (atrás de São Paulo), e o quinto com mais óbitos registrados por 100 mil habitantes, ultrapassado por Amazonas, Rondônia, Mato Grosso e o Distrito Federal (CONASS, 2021). As principais estratégias preconizadas, no país, para retardar a expansão da doença e permitir a adequação dos sistemas de saúde ao rápido aumento da demanda por leitos de internação, especialmente aqueles de terapia intensiva, evitando o colapso da assistência hospitalar, têm sido medidas de isolamento e distanciamento social, obrigatoriedade do uso de máscaras em espaços públicos e, mais recentemente, a campanha nacional de vacinação.

Em Campos dos Goytacazes, a primeira infeção pelo novo coronavírus foi confirmada em 23 de março de 2020. A partir de então, todas as ações e medidas governamentais voltaramse para o enfrentamento da pandemia. O segundo decreto municipal referente a ela, o n. 27 , de 18 de março de 2020, suspendeu o expediente externo e o atendimento presencial no âmbito da prefeitura, inclusive o das Unidades Básicas de Saúde (UBS) (PREFEITURA DE CAMPOS, 2020). Dessa forma, os objetivos, as diretrizes e as metas estabelecidos no PMS para compor as propostas e ações da cidade no período 2018-2021 foram "deixados de lado" para o enfrentamento da pandemia. Certamente, em diversos países do mundo, a resposta sanitária tem sido centrada nos serviços hospitalares, com ações para a ampliação do número de leitos, especialmente de unidades de tratamento intensivo e de respiradores pulmonares. Sem retirar, porém, a importância da adequada estruturação da atenção especializada de alta complexidade, voltada aos casos mais graves da Covid-19 e conforme preconizado pelo SUS, a atenção primária à saúde (APS) também deveria ter formado parte da estratégia geral de enfrentamento da doença.

Até fins de maio de 2021, o município em questão ocupava o sexto lugar no estado do Rio de Janeiro, em relação ao número acumulado de óbitos, com um total de 1.253 mortes 
(CONGRESSO EM FOCO, 2021), ultrapassado por Rio de Janeiro, São Gonçalo, Niterói, Nova Iguaçu e Duque de Caxias; e o $38^{\circ}$ lugar considerando o número acumulado de óbitos por 100 mil habitantes (MINISTÉRIO DA SAÚDE, 2021).

\section{A atenção básica em Campos dos Goytacazes, no contexto da pandemia do novo coronavírus}

Após apesentar aspectos sobressalentes do contexto econômico, político e social de Campos nos últimos anos, e respostas implementadas para o enfrentamento da Covid-19 na cidade, nesta seção ensaia-se uma reflexão sobre a gestão da atenção básica no município, durante a pandemia do novo coronavírus. Para tanto, em primeiro lugar, faz-se uma breve recapitulação da história da atenção básica no Brasil, com foco nos últimos trinta anos. Em seguida, destacam-se marcos históricos da atenção básica em Campos. Posteriormente, analisa-se qual a proposta - as diretrizes, objetivos e metas - para a atenção básica contida no Plano Municipal de Saúde 2018-2021, e, por último, contrastam-se os propósitos do Plano com o funcionamento desse nível de atenção em Campos, durante a pandemia.

\subsection{O marco mais amplo da Política Nacional de Atenção Básica}

A APS tem uma longa história no Brasil, no entanto foi na década de 1990 que sua tematização entrou na agenda da saúde (CASTRO; FAUSTO, 2012). Essa forma de assistência só passou a ser reconhecida como uma política de Estado a partir da criação, em 1994, do Programa Saúde da Família (PSF) (ANDERSON, 2019), a partir do qual a atenção básica ganhou destaque na agenda decisória do governo, materializando-se em instituições e políticas para implementá-la e desenvolvê-la.

Na época da implantação do SUS, a APS existia apenas nos postos de saúde, prestando serviços geralmente vinculados a pacotes assistenciais e apontados para alguns problemas de saúde determinados, além de práticas básicas de pré-natal, puericultura e vacinação. A assistência à saúde era dominada por uma racionalidade biomédica e hospitalocêntrica (com foco nos níveis secundário e terciário de atenção), mais alinhada com os interesses privados que atuavam no setor (ANDERSON, 2019).

A implementação do PSF foi um marco histórico importante na trajetória da APS no país, pois promoveu mudanças relevantes no modelo de atenção, na reorganização dos sistemas de saúde municipais e no financiamento da política. Sua origem é diretamente ligada ao Programa de Agentes Comunitários de Saúde (Pacs), que havia sido criado em 1991, para contribuir para a redução da mortalidade infantil e materna, principalmente nas áreas mais pobres das regiões Norte e Nordeste do país. O PSF preconizava a formação de equipes multiprofissionais, geralmente constituídas por médicos, enfermeiros, auxiliares de enfermagem e agentes comunitários de saúde, para atuarem em território de abrangência definida (CASTRO; FAUSTO, 2012). Em 1995, foi adotado como projeto prioritário do governo de Fernando Henrique Cardoso (PSDB, 1995-2002) e passou a ser cada vez mais destacado pelo Ministério da Saúde como "[...] a principal estratégia para a estruturação dos sistemas municipais de saúde a partir da atenção primária, visando reorientar o modelo assistencial e imprimir uma nova dinâmica na organização dos serviços e ações de saúde.” (CASTRO; FAUSTO, 2012, p. 178). A 
Norma Operacional Básica de 1996 (NOB 96) (BRASIL, 1996) contribuiu ao fortalecimento da APS, a partir da criação do Piso de Atenção Básica (PAB), com um componente fixo, calculado em base per capita, e um componente variável, cujo principal incentivo se vinculava ao PSF (CASTRO; FAUSTO, 2012).

Entre 2005 e 2012, período transcorrido durante os governos de Luiz Inácio Lula da Silva (PT, 2003-2010), no qual, ademais, havia um movimento de reafirmação da PSF como elemento central da política de APS no interior do Ministério de Saúde (CASTRO; FAUSTO, 2012), houve intensa implementação de programas de fortalecimento, qualificação e expansão do PSF, renomeado, mais tarde, para Estratégia Saúde da Família (ESF), sendo vários deles no marco da Secretaria de Gestão do Trabalho e da Educação na Saúde (SDTES), que havia sido criada em 2003 e vinculada ao Ministério da Saúde (ANDERSON, 2019).

Outro marco importante no processo de consolidação nacional da APS ocorreu com a Política Nacional de Atenção Básica (Pnab), implementada em 2006 e que integrou inúmeras portarias fragmentadas que regiam esse nível do sistema. A Pnab converteu o PSF em ESF, passando a considerar como fundamental o apoio do nível federal na formação de pessoas para trabalhar na atenção básica (ANDERSON, 2019).

Dois anos depois, em 2008, foram criados os Núcleos de Apoio à Saúde da Família (Nasf), integrados por trabalhadores da Saúde e de outras áreas e especialidades diferentes dos presentes na equipe multiprofissional básica da ESF, tradicionalmente formada por médicos generalistas, enfermeiros, auxiliares de enfermagem e agentes comunitários de saúde. Por meio da ampliação do leque de especialistas atuando na atenção básica, como psicólogos, nutricionistas e assistentes sociais, os Nasf buscavam fortalecer as equipes, suas possibilidades de intervenção e a resolutividade de suas ações. Mais tarde, a Pnab de 2011 reforçou a incorporação desses Núcleos nas equipes (ANDERSON, 2019).

Um outro marco histórico da APS no Brasil ocorreu durante o governo de Dilma Rousseff (PT, 2011-2016), com a criação do Programa Mais Médicos (PMM), no ano de 2013. O PMM visava resolver um problema crônico da saúde no Brasil: a alocação de profissionais para a prática da APS em municípios remotos e nas periferias dos grandes centros urbanos. Outros modelos implantados especificamente para o provimento e fixação de médicos nas áreas com maior percentual de pobreza, de localização remota e de difícil acesso; ou com populações em maior vulnerabilidade não haviam conseguido suprir a demanda dos municípios. Entre os principais objetivos do PMM, estavam a consolidação da ESF; a diminuição da escassez de médicos, sobretudo de APS, em regiões remotas e vulneráveis do ponto de vista socioeconômico; e a redução de desigualdades no acesso à saúde (ANDERSON, 2019).

Apesar de apresentar resultados positivos em termos de cobertura e de indicadores de saúde, o PMM enfrentou a intensa resistência das principais entidades médicas nacionais: a Associação Médica Brasileira (AMB), a Associação Nacional de Médicos Residentes (ANMR), o Conselho Federal de Medicina (CFM) e a Federação Nacional dos Médicos (Fenam). Essas organizações articularam ações desde antes de o PMM ser implementado, chegando a recorrer à justiça federal contra sua criação e a denunciar o Programa perante a OMS e a Organização Internacional dos Trabalhadores (OIT) (SILVA, 2018).

A partir de 2016, quando a presidenta Dilma Rousseff foi afastada do cargo e substituída por seu vice, Michel Temer, iniciou-se um período de privatização e de retrocesso de direitos sociais. Na área da Saúde, o governo aprovou a Emenda Constitucional n. 95/2016 (BRASIL, 2016), que congelou os gastos do setor por 20 anos, agudizando o problema do 
subfinanciamento crônico do SUS, ao mesmo tempo em que incentivava o crescimento do setor privado de saúde (ANDERSON, 2019). Essa medida retirou 20 bilhões de reais do SUS em 2019, e a projeção do Centro Brasileiro de Estudos de Saúde (Cebes) é de que a perda seja de 168 bilhões de reais até 2036 (MONTEIRO, 2020).

Nesse contexto, a APS também sofreu impactos negativos. Importantes organizações de estudos de saúde coletiva e defesa do SUS criticaram a Pnab de 2017, avaliando que essa anulava a prioridade tradicionalmente dada à ESF, em um contexto de diminuição de recursos. A nova Pnab permitiu maior flexibilização na implementação de modelos de APS, relativizando o caráter central da ESF e redefinindo alguns dos seus princípios, ocasionando retrocessos na cobertura, no padrão de serviços, no processo de trabalho e na integralidade dos cuidados (ANDERSON, 2019).

As políticas de saúde do governo de Jair Bolsonaro parecem ir na mesma direção imprimida por seu antecessor: seguem o caminho do subfinanciamento e da privatização. No âmbito da APS, foi lançado, em 2019, o Programa Saúde na Hora, que propõe a transformação das Unidades de Saúde da Família em unidades de pronto-atendimento nos municípios de médio e grande porte, apontando para uma maior desvalorização da ESF (ANDERSON, 2019), seguindo uma tendência aberta pela Pnab de 2017.

Também em 2019, por meio da Medida Provisória n. 890 (BRASIL, 2019), o governo apresentou o Programa Médicos pelo Brasil, que busca substituir o PMM, mas trazendo alguns retrocessos em relação ao seu antecessor: não faz referência à formação por meio de residências médicas, deixa dúvidas em relação à participação das universidades no desenvolvimento da ESF e reduz drasticamente o escopo de ações da APS. Na esteira da privatização da atenção básica e do SUS, o documento também propõe a criação da Agência para o Desenvolvimento da Atenção Primária à Saúde (Adaps), que constitui “[...] serviço social autônomo na forma de pessoa jurídica de direito privado com a finalidade de promover, em âmbito nacional, a execução de políticas da atenção primária à saúde.” (BRASIL, 2019). Sua implementação não representa outra coisa que a transferência da execução de uma política pública para uma agência privada (ANDERSON, 2019).

\subsection{A Atenção Primária à Saúde em Campos dos Goytacazes}

A partir da regulamentação do SUS ocorrida no ano de 1988, baseada na universalidade, na equidade, na integralidade e nas diretrizes organizacionais de descentralização e participação social; e para diferenciar-se da concepção seletiva de APS, passou-se a usar o termo atenção básica em saúde, definido como ações individuais e coletivas situadas no primeiro nível, voltadas à promoção da saúde, à prevenção de agravos, ao tratamento e à reabilitação (PONTES, 2009).

O espaço de consolidação da política de acesso ao SUS é delimitado levando-se em conta a APS, sendo os problemas de saúde, na prática, referenciados pelo nível primário da atenção, ou seja, pelas UBSs, garantindo o preceito legal da descentralização dos serviços nos municípios e tendo-se como "porta de entrada" do sistema de saúde as referidas unidades (GIOVANELLA, 2006).

Como visto, em 1994, foi criado, no Brasil, o PSF, cujo principal objetivo era a reorientação do modelo de atenção à saúde a partir do incentivo ao acesso aos serviços de saúde por meio da "porta de entrada" da APS. O PSF foi inicialmente voltado para estender a cobertura assistencial em áreas de maior risco social, mas, aos poucos, adquiriu centralidade na 
agenda governamental. "Desde 1999, passou a ser considerado pelo Ministério da Saúde como uma estratégia estruturante dos sistemas municipais de saúde, com vistas a reorientar o modelo assistencial e imprimir uma nova dinâmica na organização dos serviços e ações de saúde." (SILVA, 2012, p. 51).

No município de Campos dos Goytacazes, o PSF teve início em 1999, a partir do Centro Saúde Escola de Custodópolis, vinculado à Faculdade de Medicina de Campos (FMC) e onde passaram a funcionar três equipes de Saúde da Família. Nesse mesmo ano, foram implantados 15 grupos na cidade, número que foi progressivamente crescendo até alcançar a quantidade de 27 em 2001, cobrindo 16,82\% da população (SILVA, 2012). A partir da implantação do PSF, foi possível observar significativa mudança no perfil epidemiológico do município em questão, com efeitos positivos sobre a mortalidade infantil, neonatal e pós-neonatal (SILVA, 2012).

No ano de 2007, o PSF foi interrompido no município. Houve perda significativa na organização da atenção à saúde, fechando-se 27 Unidades de Saúde da Família, de um total de 69. Provocou-se um congestionamento de atendimentos em unidades de emergência e nas UBS restantes, as quais se mostraram insuficientes para acompanhar a demanda dos bairros e localidades, desfavorecendo a política de descentralização prevista pelo SUS. Os atendimentos que inicialmente seriam sensíveis aos recursos do nível primário da atenção começaram a ser recebidos nas grandes emergências municipais, deslocando-se $o$ foco da atenção (ALFRADIQUE, 2009).

$\mathrm{Na}$ visão dos gestores municipais à época, no entanto, Campos contava com uma atenção primária estruturada, a partir da substituição do PSF por outras ações, como o Programa de Assistência aos Assentamentos, Acampamentos e Quilombolas (Paaq), o Programa Saúde na Escola (PSE), o programa Emergência em Casa e as Unidades Básicas de Saúde 12 horas e 24 horas (SILVA, 2012). Na perspectiva de Silva (2012), a modificação da estrutura do nível primário, marcada pela ausência do PSF e pela introdução de políticas substitutivas ou compensatórias deste, trouxe maus resultados e problemas relacionados ao acesso à saúde, apontando para um aumento no número de internações hospitalares por condições sensíveis à atenção primária. A presença desse tipo de ocorrência sugere a existência de barreiras de acesso ao primeiro nível de atenção ou a outros serviços de saúde, na medida em que decorrem de doenças - como as de origem circulatória, respiratória ou do trato geniturinário - que podem ser alvo de uma ação bem planejada, por meio de uma política de atenção básica atuante e preventiva. Quando essa influência positiva ocorre, a atenção primária reduz as internações por complicações agudas da enfermidade, as readmissões e o tempo de permanência no hospital (ALFRADIQUE, 2009).

A partir de $1 .^{\circ}$ de outubro de 2009 , a gestão da saúde do município teve uma importante mudança no modelo de gerenciamento dos recursos: passou de Gestão Plena da Atenção Básica - na qual havia autonomia limitada para destinar as verbas da saúde - para a Gestão Plena Municipal - com autonomia plena na alocação dos recursos da saúde da cidade. Esse fato conferiu maior poder decisório aos gestores municipais para a utilização das verbas da Saúde, particularmente em relação à APS (MELAMED; COSTA, 2003).

Silva (2012) observava, na época da produção de sua pesquisa, uma forte tendência no aumento de recursos financeiros destinados à média e à alta complexidade em Campos dos Goytacazes, em sentido contrário à política de saúde preconizada pelo Ministério da Saúde, que enfatizava a importância do nível primário de atenção. Foi assinada uma série de convênios que significaram o repasse de verbas públicas destinadas à Saúde para a realização de exames 
diagnósticos, de média e alta complexidade, fortalecendo, dessa forma, os níveis secundários e terciários de assistência. A Gestão Plena Municipal também simplificou o processo de credenciamento de hospitais.

Segundo o Plano Municipal de Saúde de Campos dos Goytacazes 2018-2021, Campos conta com 73 UBSs, dentre as quais 17 possuem equipe da ESF ${ }^{1}$. Essa, com a participação dos Agentes Comunitários de Saúde (ACS) e das Equipes de Saúde Bucal (ESB), pretende superar o antigo modelo, exclusivamente centrado na doença, passando a uma ação preventiva, que deverá sempre se integrar a todo o contexto de reorganização do sistema de saúde (PREFEITURA DE CAMPOS, 2018a).

\subsection{Quais são as propostas do PMS de Campos para a atenção básica?}

O PMS é um instrumento básico de planejamento do SUS, que serve para a gestão da saúde pública no âmbito municipal. Propõe-se à identificação das necessidades em Saúde da população do território específico do município (NASCIMENTO; EGRY, 2017) e da capacidade de oferta pública de ações, serviços e produtos para o seu atendimento. Ele faz o diagnóstico da situação de saúde e explicita as propostas de intervenção do governo municipal para um período de quatro anos (BRASIL, 2006).

O Plano materializa-se em um documento que sistematiza o conjunto de proposições políticas na área de Saúde, ou seja, as propostas de ação relativas aos problemas e às necessidades de saúde da população do município, considerando os princípios e as diretrizes gerais que regem a política de saúde no âmbito nacional e estadual. O PMS configura, dessa forma, um instrumento que apresenta as intenções e os resultados buscados no período de quatro anos e que se expressam em objetivos, diretrizes e metas (BAHIA, 2009).

O Plano Municipal de Saúde de Campos dos Goytacazes 2018-2021 foi elaborado para orientar a política de saúde durante o governo do prefeito Rafael Diniz (Cidadania), cujo mandato se estendeu entre 2017 e 2020. A atenção básica é um dos alvos prioritários do Plano, que declara ter por objetivos principais

[...] a qualificação permanente do Sistema Único de Saúde e [...] a [garantia de] uma Atenção Básica de qualidade para a população de Campos, articulando as atividades de detecção precoce de agravos, fatores de risco e doenças com o processo de vinculação e assistência contínua e coordenada; além de garantir oferta de atenção especializada de forma descentralizada, resolutiva e articulada com Atenção Básica (PREFEITURA DE CAMPOS, 2018a, p. 88).

O alcance desses compromissos, no período 2018-2021, é orientado por dois eixos, quatro diretrizes e cinco objetivos, a serem detalhados a seguir.

O primeiro eixo refere-se ao direito à saúde, à garantia de acesso, à atenção de qualidade e à participação social. Tem como primeira diretriz a ampliação e a qualificação do acesso aos serviços de saúde "[...] em tempo adequado, com ênfase na humanização, equidade e no atendimento das necessidades de saúde, aprimorando a política de atenção básica e especializada, ambulatorial e hospitalar." (PREFEITURA DE CAMPOS, 2018a, p. 89). A diretriz é desagregada em dois objetivos, que buscam:

1A ESF foi reimplantada em Campos dos Goytacazes, no ano de 2015 (MEDEIROS; CAETANO, 2017). 
a) "[...] efetivar a Atenção Básica como porta de entrada preferencial do sistema de saúde e ordenadora do cuidado na Rede de Atenção à Saúde.” (PREFEITURA DE CAMPOS, 2018a, p. 89), com metas que incluem: a implementação da ESF, com a estrutura física e equipamentos adequados; o desenho da rede básica de farmácia; o fortalecimento da rede municipal de Atenção Básica, por meio da recuperação física e ampliação das UBSs; atualização tecnológica e renovação do mobiliário; e a implementação de ações de prevenção do câncer de colo de útero, de mama e de próstata; e

b) “[...] ampliar e qualificar o acesso aos serviços de saúde, aprimorando a política de atenção básica e especializada, ambulatorial e hospitalar, respeitando a política de humanização do SUS e promovendo o cuidado integral às pessoas nos ciclos de vida" (PREFEITURA DE CAMPOS, 2018a, p. 89-90), com metas que se propõem à implementação, ao fortalecimento ou ao aprimoramento de diversos programas, além da ampliação do acesso da população a medicamentos, incentivando o uso racional e qualificado da assistência farmacêutica no âmbito do SUS.

A segunda diretriz do primeiro eixo busca consolidar as instâncias do Controle Social, garantindo a transparência e a participação cidadã e tendo como metas o fortalecimento do Conselho Municipal de Saúde e a reestruturação da Ouvidoria Municipal do SUS.

O segundo eixo do Plano - relativo à valorização do trabalho e da educação em saúde, ao financiamento do SUS e à relação público-privado - estabelece, como primeira diretriz, a promoção "[...] [da] formação, a educação permanente, a qualificação, a valorização dos trabalhadores, a desprecarização e a democratização das relações de trabalho" (PREFEITURA DE CAMPOS, 2018a, p. 92), que se buscam alcançar a partir da estruturação da política de gestão de pessoas e do aprimoramento da educação. A segunda diretriz procura "[...] garantir o financiamento sustentável para o SUS, melhorando o padrão do gasto e qualificando o financiamento com definição de áreas de atuação dos serviços." (PREFEITURA DE CAMPOS, 2018a, p. 92), tendo como objetivo o aprimoramento da "[...] gestão municipal do SUS, especialmente por meio da formulação de políticas, da qualificação dos investimentos, da indução dos resultados, da modernização administrativa e tecnológica, da qualificação e transparência da informação." (PREFEITURA DE CAMPOS, 2018a, p. 92). As metas, nesse sentido, estão dirigidas ao "[...] aprimoramento do complexo regulatório, [à] implementação dos instrumentos de monitoramento e gestão do SUS e [à] manutenção do sistema de informática e informação em saúde, através da contratação de firma especializada" (PREFEITURA DE CAMPOS, 2018a, p. 92).

O PMS de Campos em questão contém objetivos definidos em relação à atenção básica e que buscam seu aprimoramento e sua efetivação como porta de entrada e organizadora do cuidado na rede de atenção. Além disso, propõe-se à implementação da união básica de farmácia e da ESF no município, acompanhada de estrutura física e de equipamentos adequados, bem como de atualização tecnológica.

\subsection{A atenção primária à saúde em Campos dos Goytacazes, no contexto da pandemia da Covid-19}

O SUS tem, como princípio doutrinário, a cobertura estendida a todos os cidadãos, organizada a partir da APS; e a garantia, de forma ampla e aberta, ao acesso para todas as 
pessoas, famílias e comunidades a serviços e ações de promoção e proteção da saúde e de prevenção de doenças.

Como apontado em Medina et al. (2020), a atuação da APS no enfrentamento da Covid19 tem papel importante no cuidado dos casos leves, podendo ser realizada em quatro eixos: vigilância em saúde nos territórios, atenção aos usuários infectados, suporte social a grupos vulneráveis e continuidade das ações próprias da APS.

O enfrentamento à pandemia exige a elaboração de planos de gerenciamento de risco em vários níveis (nacional, estadual, municipal e local), fortalecendo a atuação no território, que considere: a população a ser acompanhada (casos leves de COVID-19 e outros problemas de saúde); a adequada proteção dos profissionais de saúde, com condição segura à realização do seu trabalho, evitando, também, que sirvam de fonte de contaminação; as mudanças organizacionais compatíveis com a realidade local; as necessidades de apoio logístico e operacional (incluindo transporte, material e equipamentos de segurança e proteção); formação e educação permanente dos profissionais de saúde; mapeamento de potencialidades e dificuldades de cada território; a retaguarda necessária a uma ação coordenada da APS com outras instituições e serviços de saúde no território de abrangência das equipes ou fora dele; e parcerias com as organizações comunitárias, potencializando habilidades e estimulando a solidariedade (MEDINA et al., 2020, p. 1-2).

Com relação à vigilância no território e ao gerenciamento do risco da pandemia, a APS também se constitui como função necessária: a partir de ações intersetoriais junto à vigilância em Saúde do município, pode colaborar qualificando as ações nessa área, envolvendo a notificação, a detecção e o acompanhamento dos casos de isolamento domiciliar.

O trabalho dos ACSs também é necessário no enfrentamento da pandemia, especialmente no que diz respeito à conscientização da população, ao combate ao estigma relacionado à doença, à difusão de informações corretas sobre a prevenção da Covid-19 sobretudo considerando a intensa circulação de fake news - e ao apoio às atividades educativas no território, com dicas de cuidado e higiene.

Medina et al. (2020) apontam que, na atenção aos usuários com Covid-19, a APS pode, a partir da organização de fluxos distintos,

[...] realizar o cuidado dos pacientes com quadros leves, separar os sintomáticos respiratórios dos usuários com outros problemas que necessitam de atenção presencial, identificando e orientando indivíduos com maior risco de desenvolver quadros graves e também garantindo o encaminhamento oportuno daqueles que necessitam de cuidados de outros níveis de atenção (MEDINA et al., 2020, p. 2).

Para que a APS, no entanto, possa ser efetiva no cuidado aos usuários, precisa contar com: recursos adequados que garantam a qualidade dos serviços; segurança do usuário e resolubilidade de problemas; garantia de disponibilidade de equipamentos de proteção individual (EPI); e instrumentos adequados para que os serviços de APS estejam integrados à rede emergencial, hospitalar e de transporte sanitário e associados à regulação de leitos com definição de fluxos e canais de comunicação abertos e ágeis, para a garantia de cuidado oportuno, 
conforme a gravidade. São ajustes necessários, pois a atuação sem a integralidade do modelo de atenção previsto no SUS acaba sendo pouco resolutiva.

A ESF pode ajudar na identificação de pessoas e de grupos vulneráveis, como idosos e sujeitos em risco, que vivam situações de abandono, por vezes agravadas com a pandemia, favorecendo a articulação com a rede de proteção social. A atuação das equipes que compõem a ESF é importante na busca das melhores soluções, de forma intersetorial, para os problemas graves e diversos dos grupos populacionais mais necessitados, que envolvem uma ação coordenada no território. A partir da identificação de pessoas em vulnerabilidade social, podem surgir demandas de abrigo para idosos, doentes crônicos solitários, mulheres vítimas de violência e população de rua, sobretudo no apoio domiciliar para idosos que não possuem vínculos familiares e que terão dificuldades de se manterem e de se cuidarem.

Apesar de a APS estar prevista no SUS como modelo de atenção prioritário e organizador do sistema, o pacto federativo definido na Constituição Federal de 1988 (BRASIL, 1988) concede soberania política aos governos locais, que podem tomar decisões de forma autônoma. Dessa forma, apesar de contar com uma rede de APS que poderia cumprir sua função de atenção e cuidado no território de Campos dos Goytacazes, que conta com 73 UBSs, a opção da gestão local, desde promulgação do início da pandemia até dezembro de 2020, foi o fechamento das Unidades e de alguns ambulatórios, com transferência dos profissionais de saúde para o atendimento centralizado. Não somente as UBSs foram fechadas nesse período: o próprio Conselho de Saúde não teve suas reuniões realizadas periodicamente, nem mesmo de forma remota.

A experiência no município aponta que o modelo de combate à Covid-19 foi a centralização, a partir da criação do Centro de Controle e Combate ao Coronavírus de Campos (CCC), um núcleo de referência especializado para atendimento aos casos confirmados ou suspeitos da doença. A criação do CCC ocorreu a partir do fechamento das UBSs, com remanejamento dos médicos e profissionais de saúde. Durante a pandemia, a população teve acesso unicamente às Unidades Pré-hospitalares de Urgência (UPH) e ao CCC, pois todos os ambulatórios foram fechados.

A opção da gestão de fechar as UBSs trouxe impactos não somente para se alcançar o fim da pandemia. Sendo a Saúde considerada serviço essencial, os serviços especializados, bem como o acompanhamento ambulatorial de usuários com doenças crônicas, não deveriam ter sido fechados, mas, sim, continuados, com adoção de tecnologias da informação e comunicação (TIC), por meio do telefone e da internet, garantindo, assim, a oferta de ações de forma segura e sem descontinuidade, considerando que a proposta era o distanciamento social, mas não a ausência de atenção e cuidado com saúde. As demandas frequentes de usuários em acompanhamento de doenças crônicas, como a renovação de receitas e a busca por medicamentos, foram prejudicadas. O fechamento das UBSs também sobrecarregou a média e a alta complexidade, com aumento da demanda de usuários com doenças crônicas em crise, como hipertensão e diabetes, por falta de cuidado ambulatorial.

Em decorrência do fracasso da inauguração do hospital de campanha financiado pelo estado do Rio de Janeiro, a ampliação de leitos hospitalares era certamente uma necessidade da gestão, principalmente em um país desigual como o Brasil, onde há uma média de 1,98 leitos para cada mil habitantes (MEDINA et al., 2020), contrastando com o valor mínimo preconizado pela OMS, de três leitos para 1000 pessoas. Se o problema, no entanto, era a falta de leitos 
suficientes, também era necessário investir na diminuição de internações hospitalares, um dos objetivos específicos da APS e da ESF, conforme foi apresentado.

As atividades de rotina da APS precisam ser preservadas em tempos de pandemia, considerando que as previsões apontam para um longo curso de convivência com o novo coronavírus, com alternância de períodos de maior e menor isolamento social, o que exige readequação de certos procedimentos e incorporação de outros para que a APS funcione cumprindo sua missão, incluindo novas formas de cuidado cotidiano à distância e evitando o risco de aprofundamento da exclusão do acesso e das desigualdades sociais.

Ressaltamos também que a decisão de fechamento das UBSs não foi objeto de discussão no Conselho Municipal de Saúde, pois, durante a pandemia, não foi identificada nenhuma pauta convocada pelo órgão de controle, indo na contramão das deliberações do PMS.

\section{Considerações finais}

Desde sua criação, pela Constituição Federal de 1988, o SUS compõe a política de seguridade social brasileira e vem desenvolvendo sua institucionalização orientado por princípios e diretrizes inscritos na Carta Magna. Seu princípio universal de salvar vidas de forma igualitária é, para a OMS, a melhor forma de se enfrentar a pandemia. Nota-se, no entanto, que, desde a promulgação do referido código legislativo, o caráter público do SUS e o acesso amplo a ele são afetados pelo receituário neoliberal aplicado em todos os países da América Latina, que tem, como princípios fundamentais, a redução da intervenção estatal e a primazia do mercado, trazendo, em forma de proposta, a redução de investimentos do fundo público para destiná-los ao pagamento de juros, ocasionando um desmonte gradual do SUS. Destaca-se, nesse processo, a aprovação da Emenda Constitucional n. 95 de 2016, que promulgou o congelamento, por 20 anos, dos gastos com os serviços públicos de Educação e Saúde.

A partir da pandemia da Covid-19, a centralidade do SUS e a necessidade da oferta de cobertura universal no cuidado da saúde dos brasileiros ganharam espaço nos debates e nas discussões da agenda pública nacional, com a defesa por maior volume de investimentos e por um papel mais interventor do Estado, sobretudo no nível federal. O SUS possui princípios e diretrizes que cabem ao governo nacional comandar, fator que implica planejar, coordenar, executar e avaliar ações e operações para todo o Brasil. Nota-se, porém, na contramão desse modelo de governança, instituído pelo pacto federativo na Constituição de 1988, que o governo federal vem implementando uma estratégia omissa e conflitante, o que evidencia uma descoordenação nacional no combate à doença.

Nesse contexto, além da enorme crise sanitária e econômica provocada pela pandemia no país, ainda foi acrescentada a crise política ocasionada pela ausência do comando nacional e por ações deliberadas de propagação da doença por parte do governo federal (VENTURA; AITH; REIS, 2021). Como apontam Senna et al. (2021), o Brasil tem sido reconhecido internacionalmente como um dos piores exemplos de governança da crise provocada pelo vírus e o lugar onde o negacionismo e a polarização política, amplificados pelas posturas do presidente e do alto escalão do governo, além dos constantes ataques do Poder Executivo às esferas legislativa e judiciária, bem como às autoridades dos níveis subnacionais, têm sido alguns dos entraves à adoção de medidas mais efetivas de luta contra a Covid-19. 
O presente capítulo buscou trazer uma reflexão sobre a atenção básica em Campos dos Goytacazes, RJ, no contexto da pandemia do novo coronavírus, a partir da análise das diretrizes, dos objetivos e das metas do PMS da cidade, considerando o período 2018-2021, definido pelo governo do prefeito Rafael Diniz (Cidadania), cujo mandato se estendeu entre 2017 e 2020 - o primeiro ano de pandemia.

Considera-se que, em sentido contrário aos objetivos do Plano para o período 2018-2020, a atenção básica em Campos não só não foi aprimorada, como também se tornou excluída da rede de atenção durante a crise da Covid-19. Podendo contribuir na prevenção e no acompanhamento da doença, bem como na descompressão da demanda por assistência hospitalar, a atenção básica foi deixada de lado como estratégia de cuidado, comprometendo, inclusive, o monitoramento de outros agravos que normalmente são atendidos no nível primário. Para o combate à doença na cidade, o poder público deu prioridade à média e à alta complexidade, seguindo uma tendência de longa data no município, de fortalecimento dos níveis secundários e terciários de assistência à saúde, em detrimento do primário.

Após 15 meses desde o decreto municipal que suspendeu o expediente externo e o atendimento presencial no âmbito da prefeitura, apenas 30 das 73 UBSs existentes na cidade voltaram a funcionar (PREFEITURA DE CAMPOS, 2021). Além de obstaculizar tarefas de prevenção, acompanhamento e atendimento de casos de Covid-19 de sintomatologia leve; e de interromper os cuidados de pacientes com doenças crônicas, o funcionamento parcial da atenção básica transfere demanda para o já sobrecarregado nível hospitalar e não intervém nas sequelas da Covid-19 apresentadas por pessoas recuperadas, algumas das quais são passíveis de serem tratadas no nível primário de atenção.

O funcionamento parcial da atenção básica também acaba afetando o cumprimento de um dos eixos centrais do PMS, referido, entre outros aspectos, ao direito à saúde e à garantia de acesso a recursos hospitalares, de modo que o fechamento das UBSs gera mais um obstáculo para esse processo.

A situação de crise sanitária, social e econômica provocada pela pandemia de Covid-19 e a necessidade de ações de proteção social e cuidado da saúde colocaram em xeque os discursos e as práticas adotados pelas medidas de ajuste fiscal do modelo econômico do governo do atual presidente, Jair Bolsonaro. O Ministério da Saúde precisa protagonizar a coordenação e a execução da política de saúde em todo o território do país; para tanto, é necessário que o SUS não continue sendo asfixiado com ajustes fiscais e emendas constitucionais que contingenciam os recursos destinados pela União.

O sucesso da luta contra a Covid-19 e o futuro do SUS dependem de uma outra lógica de utilização do fundo público em todos os âmbitos - federal, estadual e municipal - que amplie recursos com foco na qualidade dos serviços prestados à população. É necessário um SUS fortalecido e respeitado como patrimônio da sociedade brasileira. A atenção básica precisa ser enrijecida e estruturada para a garantia do acesso à saúde, fazendo ecoar o grito dos movimentos populares dessa área: saúde é direito, não mercadoria! Vida longa ao SUS! 


\section{Referências}

ANDERSON, M. I. P. Médicos pelo Brasil e as políticas de saúde para a Estratégia Saúde da Família de 1994 a 2019: caminhos e descaminhos da Atenção Primária no Brasil. Rev. Bras. Med. Fam. Comunidade, Rio de Janeiro, v. 14, n. 41, p. 1-16, 2019. Disponível em: https://www.rbmfc.org.br/rbmfc/article/view/2180/998. Acesso em: 19 ago. 2020.

BAHIA. Manual prático de apoio à elaboração de Planos Municipais de Saúde. Salvador: Sesab, 2009. Disponível em: http://www2.saude.ba.gov.br/arquivos/MANUAL_PARA_ELABORA\%C3\%87\%C3\%83O_D E_PMS.pdf. Acesso em: 16 jun. 2021.

BRASIL. Constituição da República Federativa do Brasil de 1988. Brasília, DF: Presidência da República, [1988]. Disponível em: http://www.planalto.gov.br/ccivil_03/constituicao/constituicao.htm. Acesso em: 17 jun. 2021.

BRASIL. Norma Operacional Básica do Sistema Único de Saúde-SUS, de 06 de novembro de 1996. Brasília, DF: MS, [1996]. Disponível em: http://conselho.saude.gov.br/legislacao/nobsus96.htm. Acesso em: 17 jun. 2021.

BRASIL. Portaria n. 3.332, de 28 de dezembro de 2006. Aprova orientações gerais relativas aos instrumentos do Sistema de Planejamento do SUS. Brasília, DF: MS, [2006]. Disponível em: https://bvsms.saude.gov.br/bvs/saudelegis/gm/2006/prt3332_28_12_2006.html. Acesso em: 16 jun. 2021.

BRASIL. Emenda Constitucional n. 95, de 2016. Altera o Ato das Disposições Constitucionais Transitórias, para instituir o Novo Regime Fiscal, e dá outras providências. Brasília, DF: Presidência da República, [2016]. Disponível em: https://www2.camara.leg.br/legin/fed/emecon/2016/emendaconstitucional-95-15-dezembro2016-784029-publicacaooriginal-151558-pl.html. Acesso em: 17 jun. 2021.

BRASIL. Medida Provisória n. 890, de 01 de agosto de 2019. Institui o Programa Médicos pelo Brasil e a Agência para o Desenvolvimento da Atenção Primária à Saúde. Brasília, DF: Presidência da República, [2019]. Disponível em: http://www.planalto.gov.br/ccivil_03/_ato2019-2022/2019/Mpv/mpv890.htm. Acesso em: 23 abr. 2021.

CAMPOS DOS Goytacazes, RJ. AtlasBR, [s. l.], [2021]. Disponível em: https://atlasbrasil.org.br/perfil/municipio/330100\#idhm-all. Acesso em: 9 jun. 2021.

CASTRO, A. L. B.; FAUSTO, M. C. R. A Política Brasileira de Atenção Primária à Saúde. In: MACHADO, C. V.; BAPTISTA, T. W. F.; LIMA, L. D. (org.). Políticas de Saúde no Brasil: continuidades e mudanças. Rio de Janeiro: Fiocruz, 2012. p. 173-197. 
DAUMAS, R. P. et al. O papel da atenção primária na rede de atenção à saúde no Brasil: limites e possibilidades no enfrentamento da COVID-19. Cad. Saúde Pública, Rio de Janeiro, n. 36, 2020. Disponível em:

https://www.researchgate.net/publication/342498461O_papel_da_atencao_primaria_na_rede_d e_atencao_a_saude_no_Brasil_limites_e_possibilidades_no_enfrentamento_da_COVID-19. Acesso em: 16 jun. 2021.

FARIAS, A. R. et al. Comunicado Técnico 4: identificação, mapeamento e quantificação das áreas urbanas do Brasil. Campinas, SP: Embrapa, 2017. Disponível em: https://ainfo.cnptia.embrapa.br/digital/bitstream/item/176016/1/20170522-COT-4.pdf. Acesso em: 9 jun. 2021.

GIOVANELLA, L. A atenção primária à saúde nos países da União Europeia: configurações e reformas organizacionais na década de 1990. Cad. Saúde Pública, Rio de Janeiro, v. 22, n. 5 , mai. 2006.

IBGE. Produto interno bruto dos municípios. [s. 1.]: IBGE, 2018. Disponível em: https://www.ibge.gov.br/estatisticas/economicas/contas-nacionais/9088-produto-internobruto-dos-municipios.html?=\&t=resultados. Acesso em: 9 jun. 2021.

IBGE. Áreas territoriais. [s. 1.]: IBGE, 2020a. Disponível em: https://www.ibge.gov.br/geociencias/organizacao-do-territorio/estrutura-territorial/15761areas-dos-municipios.html?t $=$ acesso-ao-produto\&c $=3304557$. Acesso em: 9 jun. 2021.

IBGE. Campos dos Goytacazes. [s. 1.]: IBGE, 2021. Disponível em: https://cidades.ibge.gov.br/brasil/rj/campos-dos-goytacazes/panorama. Acesso em: 9 jun. 2021.

IBGE. Estimativas da população. [s. 1.]: IBGE, 2021b. Disponível em: https://www.ibge.gov.br/estatisticas/sociais/populacao/9103-estimativas-depopulacao.html? $=\& \mathrm{t}=$ resultados. Acesso em: 9 jun. 2021.

LFRADIQUE, M. E. et al. Internações por condições sensíveis à atenção primária: a construção da lista brasileira como ferramenta para medir o desempenho do sistema de saúde (Projeto ICSAP - Brasil). Cad. Saúde Pública, Rio de Janeiro, v. 25, n. 6, jun. 2009. Disponível em: http://www.scielo.br/scielo.php. Acesso em: 01 nov. 2020.

MEDEIROS, J, A.; CAETANO, R. C. Saúde da Família em Campos dos Goytacazes (RJ): uma história recente. Anais do Encontro Internacional e Nacional de Política Social, v. 1, n. 1, p. 1-3, 2017. Disponível em: https://www.periodicos.ufes.br/einps/article/view/16520. Acesso em: 19 fev. 2021. 
MEDINA, M. G. et al. Atenção primária à saúde em tempos de COVID-19: o que fazer?. Cad. Saúde Pública, Rio de Janeiro, v. 36, n. 8, p. 1-5, 2020. Disponível em: http://cadernos.ensp.fiocruz.br/static//arquivo/1678-4464-csp-36-08-e00149720.pdf. Acesso em: 30 mai. 2021.

MELAMED, C.; COSTA, N. R. Inovações no financiamento federal à Atenção Básica. Cênc. saúde coletiva, Rio de Janeiro, v. 8, n. 2, 2003. Disponível em: http://www.scielo.br/scielo.php. Acesso em: 15 nov. 2020.

MINISTÉRIO DA SAÚDE. Covid-19 no Brasil. Brasília, DF: MS, [2021]. Disponível em: https://qsprod.saude.gov.br/extensions/covid-19_html/covid-19_html.html. Acesso em: 9 jun. 2021.

MONTEIRO, N. O Estado em desmonte frente à epidemia da Covid-19. Physis: Revista de Saúde Coletiva, Rio de Janeiro, v. 30, n. 3, p. 1-9, 2020. Disponível em: https://scielosp.org/pdf/physis/2020.v30n3/e300304/pt. Acesso em: 16 jun. 2021.

NASCIMENTO, A. B.; EGRY, E. Y. Os planos municipais de saúde e as potencialidades de reconhecimento das necessidades em saúde: estudo de quatro municípios brasileiros. Revista Saúde Soc. São Paulo, v. 26, n. 4, p. 861-871, 2017. Disponível em: https://www.scielosp.org/pdf/sausoc/2017.v26n4/861-871/pt. Acesso em: 4 mai. 2021.

PAINEL CONASS Covid-19. Conass, [s. l.], 2021. Disponível em: https://www.conass.org.br/painelconasscovid19/. Acesso em: 09 jun. 2021.

PAINEL COVID-19. Congresso em foco, [s. l.], [2021]. Disponível em: https://congressoemfoco.uol.com.br/covid19/index.html. Acesso em: 09 jun. 2021.

PONTES, A. P. M. et al. O Princípio de Universalidade do Acesso aos Serviços de Saúde: O que pensam os usuários? Rev. Enferm., Rio de Janeiro, v. 13, n. 3, jul. 2009. Disponível em: http://www.scielo.br/scielo.php. Acesso em: 28 dez. 2019.

PREFEITURA DE CAMPOS. Decreto n. 27, de 16 de março de 2020. Dispõe sobre as ações necessárias à redução do contágio pelo Covid-19 - coronavírus, e dá outras providências. Diário oficial do município de Campos dos Goytacazes, n. 550, 18 mar. 2020. Disponível em: https://transparencia.campos.ri.gov.br/attachments/9294fe48a937358a2e4857e54b41976f3914c 090/store/eebaf8ff296f38d8abba7553e0c84b0038c1281cc00e7cc1546d6acd67ae/Decreto+Muni cipal $+\mathrm{n} \% \mathrm{C} 2 \% \mathrm{BA}+27-2020+18+\mathrm{de}+$ mar $\% \mathrm{C} 3 \% \mathrm{~A} 7 \mathrm{o}+\mathrm{de}+2020$.pdf. Acesso em: 9 jun. 2021.

PREFEITURA DE CAMPOS DOS GOYTACAZES. Plano Municipal de Saúde de Campos dos Goytacazes 2018-2021. Campos dos Goytacazes, RJ: PMCG, 2018a. Disponível em: https://www.campos.rj.gov.br/up/pdiretor.php?id_arquivo=12. Acesso em: 19 fev. 2021.

PREFEITURA DE CAMPOS DOS GOYTACAZES. Plano Municipal de Assistência Social de Campos dos Goytacazes 2018-2021. Campos dos Goytacazes, RJ: PMCG, 2018b. 
Disponível em: https://www.campos.rj.gov.br/up/pdiretor.php?id_arquivo=11. Acesso em: 19 fev. 2021.

PREFEITURA DE CAMPOS. Unidades Básicas de Saúde em funcionamento. Campos dos Goytacazes, RJ: Secretaria Municipal de Saúde, 2021.

RAMOS, T. T. Crescimento Econômico e Desenvolvimento sócio-espacial em Campos dos Goytacazes. Geo UERJ, Rio de Janeiro, n. 29, p. 63-88, 2016. Disponível em: https:/ /www.epublicacoes.uerj.br/index.php/geouerj/article/view/16109/19559. Acesso em: 16 jun. 2021.

RODRIGUES, P. H. A.; SANTOS, I. S. Saúde e cidadania. Uma visão histórica e comparada do SUS. São Paulo: Atheneu, 2011.

RUIZ J. L. S.; SILVA, W. M. F. A centralidade do SUS na pandemia do coronavírus e as disputas com o projeto neoliberal. Physis: Revista de Saúde Coletiva, Rio de Janeiro, v. 30, n. 3, p. 1-8, 2020. Disponível em:

https://www.scielo.br/j/physis/a/5LqczYMjkxnqZXGfXfckxTK/?lang=pt\&format=pdf. Acesso em: 30 maio 2021.

SENNA, M. C. M.; SOUTO MAIOR, A.; SUÁREZ BALDO, V. S. Proteção Social em Tempos de COVID-19: experiências na América Latina. Argumentum, Vitória, v. 13, n. 1, p. 66-80, jan./abr. 2021. Disponível em: https://periodicos.ufes.br/argumentum/article/view/33033. Acesso em: 7 mai. 2021.

SILVA, A. T. M. F. A organização da política pública de saúde no Município de Campos dos Goytacazes/RJ: uma análise do acesso à Atenção Básica (2007-2011). 2012. 92 f. Dissertação (Mestrado em Políticas Sociais) - Centro de Ciências do Homem, Universidade Estadual do Norte Fluminense Darcy Ribeiro, Campos dos Goytacazes, 2012.

SILVA, L. N. Programa Mais Médicos: embates políticos entre entidades médicas e o Estado. 2018. 158 f. Dissertação (Mestrado em Saúde Coletiva) - Instituto de Medicina Social, Universidade do Estado do Rio de Janeiro, Rio de Janeiro, 2018.

VENTURA, D. F. L.; AITH, F. M. A.; REIS, R. R. (coord.). A linha do tempo da estratégia federal de disseminação da COVID-19. São Paulo: Cepedisa, UPS, 2021. Disponível em: https://cepedisa.org.br/wp-content/uploads/2021/06/CEPEDISA-USP-Linha-do-TempoMaio-2021_v3.pdf. Acesso em: 16 jun. 2021. 